\title{
Anémie hémolytique et nouveau souffle cardiaque chez un homme de 77 ans
}

\author{
Danyal Ladha MD, Karima Khamisa MD, Steven Promislow MD
}

— Citation : CMAJ 2021 September 7;193:E1389. doi : 10.1503/cmaj.202620-f

Voir la version anglaise de l'article ici : www.cmaj.ca/lookup/doi/10.1503/cmaj.202620

$\mathbf{U}$ n homme de 77 ans atteint de démence vasculaire s'est présenté au service des urgences pour de la dyspnée. Ses antécédents médicaux comprenaient une sténose mitrale rhumatismale et un remplacement de valve mitrale avec prothèse mécanique en 2001. À l'examen, le patient était afébrile et euvolémique; il présentait un ictère. À l'auscultation, un nouveau souffle holosystolique était audible, en particulier à l'apex. Les analyses de laboratoire ont révélé un faible taux d'hémoglobine (89 g/L [plage normale 125-170]), un volume globulaire moyen (98,9 fl [plage normale $80-100])$, une numération plaquettaire normale, un taux d'haptoglobine indétectable, et une élévation de la lacticodeshydrogénase (1603 U/L [plage normale 99-167]), de la bilirubine (totale : $45 \mu \mathrm{mol} / \mathrm{L}$ [normale < 15]; directe : $19 \mu \mathrm{mol} / \mathrm{L}$ [normale < 5]) et de la numération réticulocytaire $(42,9 \%$ [plage normale 1,5\%-15\%]). Le test direct à l'antiglobuline (TDA) (anciennement test de Coombs direct) était négatif. Nous avons observé de nombreux schizocytes au frottis sanguin (figure 1). Un échocardiogramme transthoracique a montré une déhiscence postérieure de la valve prothétique, accompagnée d'une régurgitation paravalvulaire mitrale substantielle absente lors de l'échocardiogramme effectué 5 mois auparavant (annexe 1 , accessible en anglais au www.cmaj.ca/lookup/doi/10.1503/ cmaj.202620/tab-related-content). La fraction d'éjection du ventricule gauche était de $50 \%$ à $55 \%$. Les hémocultures négatives, couplées à l'absence de fièvre ou d'autres indices infectieux, rendaient l'endocardite improbable. Le patient a reçu un diagnostic d'anémie hémolytique macroangiopathique causée par la déhiscence de la valve prothétique. Il a été traité de façon conservatrice étant donné son mauvais état fonctionnel de base.

On rapporte une anémie hémolytique chez jusqu'à $1 \%$ des patients avec valve prothétique ${ }^{1}$. La physiopathologie met en cause une régurgitation paravalvulaire causant des traumatismes mécaniques aux érythrocytes. L'apparition d'un ictère et d'un nouveau souffle cardiaque fait partie des caractéristiques cliniques de cette pathologie. Le frottis sanguin indique habituellement de nombreux schizocytes et une numération plaquettaire normale. Parmi les autres résultats de laboratoire, il faut compter une élévation de la lacticodeshydrogé-

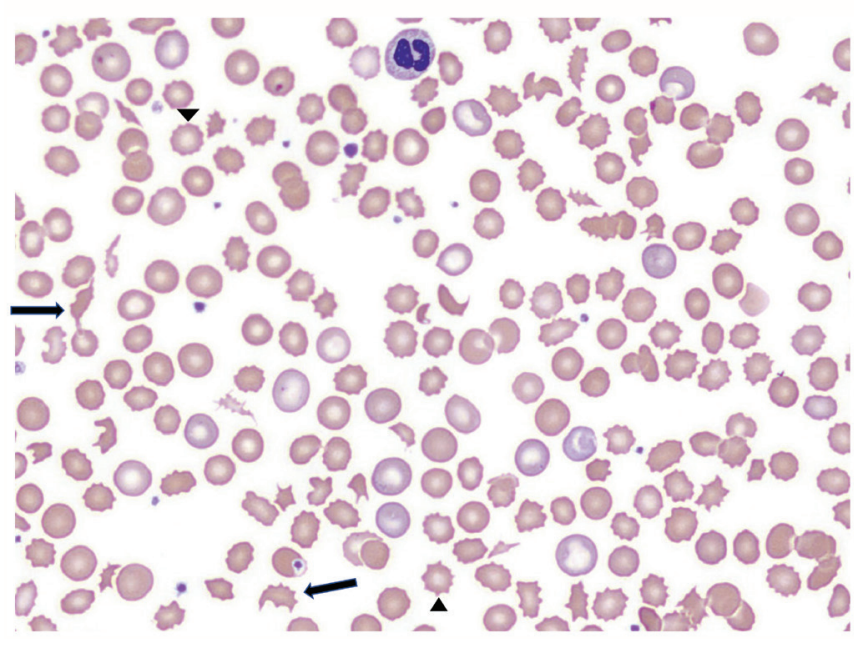

Figure 1 : Frottis sanguin d'un patient de 77 ans avec valve mitrale mécanique présentant une anémie hémolytique. Le frottis montre de nombreux schizocytes (flèches) et acanthocytes (pointes de flèches), ainsi qu'une numération plaquettaire normale.

nase, de la bilirubine et de la numération réticulocytaire, ainsi qu'un faible taux d'haptoglobine. Chez les patients avec valve prothétique qui présentent une anémie, il est important d'envisager une hémolyse associée à la valve pour le diagnostic différentiel. Les examens initiaux devraient inclure un frottis sanguin, un test direct à l'antiglobuline et une échocardiographie transthoracique pour évaluer le fonctionnement de la valve prothétique. Si les résultats pointent vers une régurgitation paravalvulaire, la prise en charge définitive sera soit une fermeture percutanée de la fuite paravalvulaire soit une réparation chirurgicale. Des mesures médicales ou d'appoint (transfusions, supplémentation en acide folique) pourraient être bénéfiques à court terme ${ }^{2}$.

\section{Références}

1. Alkhouli M, Farooq A, Go RS, et al. Cardiac prostheses-related hemolytic anemia. Clin Cardiol 2019;42:692-700.

2. Eleid MF, Cabalka AK, Malouf JF, et al. Techniques and outcomes for the treatment of paravalvular leak. Circ Cardiovasc Interv 2015;8:e001945. doi: 10.1161/ CIRCINTERVENTIONS.115.001945. 
Intérêts concurrents : Aucun déclaré.

Cet article a été révisé par des pairs.

Les auteurs ont obtenu le consentement de la famille du patient.

Affiliations : Département de médecine de l'Hôpital d'Ottawa (Ladha, Khamisa), Université d'Ottawa, Ont.; Section de la cardiologie (Promislow), Université du Manitoba, Winnipeg, Man.

Propriété intellectuelle du contenu : Il s'agit d'un article en libre accès distribué conformément aux modalités de la licence Creative Commons Attribution (CC BY-NC-ND 4.0), qui permet l'utilisation, la diffusion et la reproduction dans tout médium à la condition que la publication originale soit adéquatement citée, que l'utilisation se fasse à des fins non commerciales (c.-à-d., recherche ou éducation) et qu'aucune modification ni adaptation n'y soit apportée. Voir : https://creativecommons.org/licenses/by-nc-nd/4.0/deed.fr.

Correspondance : Danyal Ladha, dladha@toh.ca

Les images cliniques sont choisies pour leur caractère particulièrement intéressant, classique ou impressionnant. Toute soumission d'image de haute résolution claire et bien identifiée doit être accompagnée d'une légende aux fins de publication. On demande aussi une brève explication (300 mots maximum) de la portée éducative des images, et des références minimales. Le consentement écrit du patient au regard de la publication doit être obtenu avant la soumission. 